\title{
Computer scoring of the Levels of Emotional Awareness Scale
}

\author{
Kimberly A. BARCHARD \\ University of Nevada, Las Vegas, Nevada \\ JANE BAJGAR \\ University of Wollongong, Gwynneville, New South Wales, Australia \\ DUNCAN ERMini LeaF \\ Purdue University, West Lafayette, Indiana \\ AND \\ RICHARD D. LANE \\ University of Arizona, Tucson, Arizona
}

\begin{abstract}
The Levels of Emotional Awareness Scale (LEAS; Lane, Quinlan, Schwartz, Walker, \& Zeitlan, 1990) is the most commonly used measure of differentiation and complexity in the use of emotion words and is associated with important clinical outcomes. Hand scoring the LEAS is time consuming. Existing programs for scoring open-ended responses cannot mimic LEAS hand scoring. Therefore, Leaf and Barchard (2006) developed the Program for Open-Ended Scoring (POES) to score the LEAS. In this article, we report a study in which the reliability and validity of POES scoring were examined. In the study, we used three participant types (adult community members, university students, children), three LEAS versions (paper based, computer based, and the LEAS for children), and a diverse set of criterion variables. Across this variety of conditions, the four POES scoring methods had internal consistencies and validities that were comparable to hand scoring, indicating that POES scoring can be used in clinical practice and other applied settings in which hand scoring is impractical.
\end{abstract}

The Levels of Emotional Awareness Scale (LEAS; Lane, Quinlan, Schwartz, Walker, \& Zeitlan, 1990) is an open-ended test of the ability to use emotion words in a complex and differentiated fashion. Respondents describe how they and another person would feel in 20 emotionally evocative situations. Because the LEAS is open-ended, it takes a long time to score. In the first author's lab, training a new LEAS scorer can take up to $10 \mathrm{~h}$. Once trained, LEAS scorers can take up to 20 min for each respondent. Thus, a new scorer may take up to $43 \mathrm{~h}$ to score the LEAS for 100 respondents, and an experienced and efficient LEAS scorer will still take 10 min per respondent or roughly $17 \mathrm{~h}$ for 100 . The amount of time involved in scoring is likely the primary reason that researchers have not used the LEAS more often. To address this issue, Lane et al. (1990) advocated automating the LEAS. The purpose of the present project was to implement this suggestion: We developed a free computer program to score the LEAS, and in this article we assess the validity of the scores that this program produces. Before describing the scoring program, we first describe emotional awareness and the LEAS in more detail.

\section{The Levels of Emotional Awareness Scale}

Being aware of emotional experiences in the self and in others is perhaps the most fundamental of emotional intelligence skills (Lane, 2000). Individuals with higher emotional awareness have at their disposal complex and differentiated emotion information, which can be used to support higher level emotion processes such as understanding and managing emotions and using emotions to facilitate cognition.

As would be expected, lower levels of emotional awareness are related to a number of clinical conditions. LEAS scores are lower in people with somatoform disorders (Subic-Wrana et al., 2002), depression (Berthoz et al., 2000; Donges et al., 2005), eating disorders (Bydlowski et al., 2005), posttraumatic stress disorder (Frewen et al., 2008), multiple addictive behaviors (Jouanne, Edel, \& Carton, 2005), and borderline personality disorder (Levine, Marziali, \& Hood, 1997). LEAS scores increase over the course of treatment for people with somatoform disorders, independent of changes in negative affect (Subic-Wrana, Bruder, Thomas, Lane, \& Köhle, 2005), and moreover predict improvements among psychoso-

K. A. Barchard, barchard@unlv.nevada.edu 
matically ill patients (Simson, Martin, Schäfer, Franz, \& Janssen, 2006). Finally, higher scores on the LEAS are associated with a stable sense of general well-being, despite temporary negative mood (Ciarrochi, Caputi, \& Mayer, 2003). Thus, emotional awareness is central to many clinical conditions and a foundational emotional skill for all people.

There is strong evidence for the construct validity of the LEAS as a measure of emotional awareness. LEAS scores are correlated with changes in cerebral blood flow associated with film- and recall-induced emotional experiences (Frewen et al., 2008; Lane, Reiman, et al., 1998) and are related to higher accuracy in the perception of emotion (Lane, Sechrest, Riedel, Shapiro, \& Kaszniak, 2000; Lane et al., 1996; Subic-Wrana et al., 2005). In addition, several studies (Lane, Sechrest, \& Riedel, 1998; Rose, 2004; Walgren, 1996; Waller \& Scheidt, 2004) have shown that higher LEAS scores are associated with lower scores on self-reported difficulty expressing feelings.

The LEAS is based on a five-tiered developmental theory of emotional awareness (Lane \& Schwartz, 1987), modeled after Piaget's theory of cognitive development (see Flavell, 1963). In this theory, each level represents a greater degree of differentiation and integration of emotional experiences. In hand scoring, each item on the LEAS receives a score ranging from 0 (no awareness) to 5 (the highest level of awareness). See Table 1 for descriptions and examples of the six levels.

To score the LEAS by hand, the scorer needs to make a number of subjective decisions. First, the scorer needs to interpret the meaning of emotion words on the basis of context (e.g., pain can be scored as 1 or 3, depending on whether it represents physical pain or emotional pain). Second, the scorer needs to determine whether words are synonyms of each other, to decide whether a person is describing a single emotion (e.g., I would feel happy and joyous) or a blend (e.g., I would feel happy and proud). Third, the scorer needs to distinguish between emotions that are attributed to the self and emotions that are attributed to the other (e.g., I would feel loved) and determine whether the self and other are feeling the same emotions (e.g., I would be happier than my husband). These subjective decisions are fundamental to the cognitive-developmental theory on which the LEAS is based.

The subjective decisions that are involved in hand scoring cannot be precisely duplicated by a computer. The best we can hope for is to develop a computer scoring program that is relatively similar to LEAS hand scoring. Even then, the computer scoring must be considered a new method, and its validity will need to be demonstrated.

\section{Computerized Scoring}

There has been substantial work on automated scoring of open-ended responses. However, none of these methods can be adapted to mimic LEAS hand scoring. Automated essay scoring is the largest area (for reviews of specific methods and validity results, see Shermis \& Burstein, 2003; Valenti, Neri, \& Cucchiarelli, 2003; Yang, Buckendahl, Juszkiewicz, \& Bhola, 2002). These methods are typically designed to rate some combination of an essay's content and writing style. In particular, the $k$-nearest-neighbors method of Larkey (1998) could be easily adapted to score LEAS responses. This approach classifies an essay into a rating category by comparing the words contained in that essay with the words present in a collection of essays that have already been rated by hand. Unfortunately, this method would not mimic the hand scoring of the LEAS, for which we have strong validity evidence. The $k$-nearest-neighbors approach might assign high scores because of the presence of nonemotion words, such as because or $I$, and might give low scores to responses that provide detailed, clear descriptions of unusual emotional reactions.

Other computational methods have been specifically designed to measure emotional content in open-ended responses, but they cannot mimic LEAS hand scoring either. The "Dictionary of Affect in Language" (DAL; Whissell, 1989) has been used by many researchers to score text for emotional content (e.g., Dubois, 1997; Mossholder, Settoon, Harris, \& Armenakis, 1995; Whissell, 2008; Zwaal, Prkachin, Husted, \& Stones, 2003). Each word in DAL has been rated for pleasantness and activity level, on the basis of the circumplex model of emotion (Russell, 1980). To score text, DAL searches for words from its dictionary, and calculates the mean rating for the scored words for each of the two dimensions. The DAL method is similar to LEAS scoring, in that words are assigned specific scores. However, the criteria for the word scores are different: DAL is based on pleasantness and activity level, whereas LEAS hand scoring is based on the complexity and specificity of the emotion words. Furthermore, LEAS hand scoring uses criteria not specified in DAL, such as the presence of synonyms and the distinction between the self and the other. Thus, DAL could be used to score LEAS responses, but the scores would bear a limited conceptual relationship to LEAS hand scores.

The Linguistic Inquiry and Word Count program (LIWC; Pennebaker, Chung, Ireland, Gonzales, \& Booth, 2008) has also been used to score text for emotion content in a wide variety of research areas (e.g., Ali \& Levine, 2008; Carroll, 2007; Pennebaker, 1997). LIWC 2007 con-

Table 1

The Six Levels of Emotional Awareness in Hand Scoring

\begin{tabular}{cll}
\hline Level & \multicolumn{1}{c}{ Description } & \multicolumn{1}{c}{ Example Response } \\
\hline 0 & Cognitions & I would expect him to help me. \\
1 & Bodily sensations & I would feel pain. \\
2 & Action tendencies & I would cry. \\
3 & Single emotions & I would feel angry. \\
4 & Blends of emotion & I would feel happy but guilty. \\
5 & Combinations of blends & I would feel sad and frightened. My friend would feel sympathetic and relieved. \\
\hline
\end{tabular}


tains a dictionary of about 4,500 words that have been classified as falling into 1 or more of 64 linguistic categories, including several categories related to emotions. LIWC counts the number of words in a response that match the words in each linguistic category. Additionally, the LEAS glossary could be used with LIWC to obtain a count of the number of times that LEAS glossary words are used. However, LIWC could not be used to approximate LEAS hand scoring. LIWC gives the same score to each word in the list, does not take into account identical words being used repeatedly, and does not distinguish between emotions that were attributed to the self versus to the other person in the scenario.

Thus, none of the existing computerized scoring methods can be adapted to mimic LEAS hand scoring. This motivated us to write a computer program, the Program for Open-Ended Scoring (POES; Leaf \& Barchard, 2006), specifically designed for automated LEAS scoring. This program includes one scoring method (All-Sum) that is similar to those in the DAL and LIWC programs, but POES also goes beyond those methods to more closely mimic LEAS hand scoring. In addition, because POES is distributed under the GNU General Public License (Free Software Foundation, 1991), it is freely available and can be modified by others to include new scoring methods. POES consists of two files: a DOS-based program, which takes $96 \mathrm{~KB}$ of disk space, and a Windows-based interface, which takes $64 \mathrm{~KB}$ of disk space.

\section{The Present Study}

The purpose of the present project was to assess the validity of POES scoring. First, we scored three sets of data using hand scoring and POES scoring. Then, we correlated the two types of scores with each other and evaluated their internal consistencies and their correlations with criterion variables. The three data sets we used involved different types of participants and different versions of the LEAS, and the criterion variables are diverse. Therefore, any results that are consistent across the three data sets are likely to be generalizable to many other data sets.

Two of the data sets used in this article have already been used to examine the internal consistency and criterionrelated validity of the LEAS. We rescored the data from those two studies to determine whether POES scores produce similar results. First, we rescored the adult community member data from McRae, Reiman, Fort, Chen, and Lane (2008), who found that hand scoring was internally consistent $(\alpha=.84)$ and was associated with blood flow in the dorsal anterior cingulate cortex (dACC). This area is associated with awareness and monitoring of emotional experiences (Hutcherson et al., 2005; Ochsner, Bunge, Gross, \& Gabrieli, 2002; Taylor, Phan, Decker, \& Liberzon, 2003). Second, we rescored the data from Bajgar, Ciarrochi, Lane, and Deane (2005), who found that hand scoring of the children's version of the LEAS had moderate internal consistency $(\alpha=.66)$ and had a significant relationship with the Vocabulary subtest of the Wechsler Intelligence Scale for Children-Third Edition (WISC-III) (Wechsler, 1991) $(r=.31, p<.05)$ and a nearly significant relationship with emotion comprehension. The pur- pose of the present study was to determine whether POES scoring of these two data sets could replicate these internal consistencies and criterion validities.

Third, we used new research data from a computerized form of the LEAS. In the previous two data sets, we used three types of POES scores. For the third data set, we also included a fourth POES scoring method, which could only be used with the computerized form. We used adult university students to assess internal consistency and the correlations between POES scoring and hand scoring.

\section{METHOD}

Participants

\section{Sample 1: Adult Community Members}

Forty-four participants (22 female, 22 male) were recruited through advertisements posted at a medical center at a large local university and in churches, libraries, and other public facilities. The participants were screened by phone and were excluded if they reported a history of neurological abnormalities, head injury, learning disabilities, current psychoactive medication use, current drug or alcohol abuse, current major depressive episode, or lifetime prevalence of a psychotic disorder. By design, the participants ranged in age from 19 to 30 years $(M=24.6$ years, $S D=3.3)$. The participants identified themselves as follows: $75 \%$ White non-Hispanic, $14 \%$ Hispanic, $6 \%$ Asian, and 5\% African American. All of the participants spoke English as their native language. Additional information about these participants can be found in McRae et al. (2008).

\section{Sample 2: Children}

Fifty-one children ( 25 female, 26 male) were recruited from two private schools in a regional city with a population of 180,000 . They ranged in age from 10 to 11 years $(M=10.3$ years, $S D=0.48)$. All of the children came from middle-class backgrounds, with the majority of parents working in professional or semiprofessional occupations. Five children were of non-English-speaking cultural backgrounds. However, all of the children were identified as competent English speakers. Additional information about these children can be found in Bajgar et al. (2005).

\section{Sample 3: Adult University Students}

Sixty-six university students (52 female, 14 male) participated in return for course credit. They ranged in age from 18 to 46 years $(M=24.7$ years, $S D=8.1)$. The participants identified themselves as follows: $62 \%$ White/Caucasian, 9\% Black/African American, $8 \%$ Hispanic, 6\% Pacific Islander, and 6\% Asian. All of the participants either spoke English as their first language or had been speaking English for at least 10 years and reported being very comfortable reading and writing in English.

\section{Measures}

\section{LEAS}

Paper-administered LEAS. The adult community members in Sample 1 completed the original paper-administered LEAS. Each of the 20 LEAS items consists of an emotionally evocative scenario involving the reader and another person, and is presented at the top of a new page. Each scenario was designed to evoke one of four different emotions (anger, sadness, fear, happiness) at one of the five levels of emotion awareness in the Lane and Schwartz (1987) model. Each scenario is followed by two prompts: "How would you feel?" and "How would the other person feel?"

The LEAS for Children. The children in Sample 2 completed the paper-administered Levels of Emotional Awareness Scale for Children (LEAS-C; Bajgar et al., 2005). The LEAS-C consists of 12 scenarios, each involving the child and another person. Of these 12 scenarios, 10 are modified LEAS items with simplifications of 
grammar and vocabulary. Two new items reflect school and peerrelated concerns. In this study, the LEAS-C scenarios were read aloud while the children read silently. The children then provided written responses to the two questions "How would you feel?" and "How would the other person feel?"

Computer-administered LEAS. The adult students in Sample 3 completed the 20-item computer-administered LEAS. It consists of two Web pages, each containing 10 LEAS items. For each item, the scenario description was followed by two prompts: "How would you feel?" and "How would the other person feel?" After each prompt, a text input box ( 8 rows $\times 40$ columns) was used to collected the participants' responses. These Web pages were uploaded to the university server so that they could be accessed from university computer labs via the Internet.

Scoring. All three versions of the LEAS were scored in two ways. First, responses were scored using the hand-scoring method described in the manual for the adult version of the LEAS (Lane, 1991). Second, responses were spell-checked and then scored using POES Version 1.2.2 (Leaf \& Barchard, 2006) and LEAS Wordlist 2.1 (Barchard, 2006).

Hand scoring. When hand scoring the LEAS, item scores are calculated in three stages (Lane, 1991). First, each word in the response is assigned a score. Nonemotion words (e.g., expect) are scored 0. Physiological sensations (e.g., dizzy) are scored 1. Words that indicate emotions but also have nonemotional meanings (e.g., bad) are scored 2, as are actions related to emotions (e.g., cry) and personality traits (e.g., tolerant). Discrete emotion words (e.g., fear) are scored 3. The scoring manual glossary (Lane, 1991) lists words and phrases and their associated levels. However, the glossary is not exhaustive: The scorer often has to look for synonyms or check the rules regarding the types of words included at each level. Also, words often have more than one possible score, and the scorer must use these general scoring rules and the context to decide which score is appropriate.

The second stage is to calculate self and other scores. To do this, the scorer decides whether each emotion word is attributed to the participant (self) or to the other person. For example, in the statement I would be happy my love was reciprocated, who feels love-the participant, the other, or both? The scorer also has to decide whether emotion words are synonymous: Is love the same as care for? On the basis of these two subjective judgments, the scorer calculates the self and other scores. If the response has two nonsynonymous Level 3 emotion words attributed to the participant, the self score is 4 . If not, the self score is the maximum of the word scores for emotions attributed to the participant. The other score is calculated analogously on the basis of emotions attributed to the other person.

The third stage is to calculate the item score. If the self and other scores are both 4 and the self and other emotions are different, the item score is 5. Otherwise, it equals the maximum of the self and other scores. This results in item scores that range from 0 (no emotion words) through 5 (combinations of blends of emotions), corresponding to the six levels of emotional awareness in the Lane and Schwartz (1987) model (see Table 1). The total test scores are calculated as the sum of the item scores for the 20 items and thus range from 0 to 100 .

For each of the three samples, hand scoring was completed by experienced LEAS scorers, who had scored at least 1,000 responses before beginning scoring of these samples (e.g., they had previously scored at least 50 participants who completed all 20 items). Interrater reliability was assessed by having one additional person score the responses for each sample. In Sample 1 (adult community members) and Sample 3 (adult university students), the second scorers met the above definition of experienced scorers, and they rated all protocols. In Sample 2 (children), the second scorer was well trained, having received $5 \mathrm{~h}$ of training, including a detailed explanation of the scoring procedure and intensive trial scoring with feedback. The second scorer rated 22 of the protocols. In all samples, interrater reliability was high. In Sample 1, the Pearson correlation was .98; in Sample 2, it was .89; in Sample 3, it was .95.

POES scoring. To score the LEAS, POES 1.2.2 (Leaf \& Barchard, 2006) requires two input files: the participants' typed response data and a Wordlist file that specifies the score values to be given for specific words and phrases. Currently, there are two versions of the LEAS Wordlist. The initial version, LEAS Wordlist 1.0 (Leaf \& Barchard, 2002), was based directly on the LEAS hand-scoring manual (Lane, 1991). The major challenge in translating the LEAS glossary into the LEAS Wordlist 1.0 was handling words with multiple values. When the LEAS glossary gave only a single value for a word, that value was entered in LEAS Wordlist 1.0. In some cases, however, a glossary word has two values depending on context. For example, hurt could be Level 1 if it refers to a physical sensation or Level 3 if it describes an emotion. Because POES 1.2.2 cannot make distinctions on the basis of context, each Wordlist entry must be associated with only a single value. For multivalued glossary words, a team of experienced LEAS scorers discussed which interpretation was most common and decided which value would be used in the Wordlist. The LEAS Wordlist 1.0 contained 888 word and phrase entries. The main limitation of Wordlist 1.0 was that it only contained the exact phrasing from the LEAS glossary; it did not contain words that human raters treat as identical to glossary entries.

Wordlist 2.1 (Barchard, 2006) expanded the list of words and phrases from those that received scores in Wordlist 1.0. A team of five expert LEAS scorers considered 467 possible additions and modifications. The changes fell into five categories. First, if an LEAS glossary entry included a possessive determiner (e.g., at the end of my rope), additional entries were added for all remaining possessive determiners (e.g., at the end of his rope). Second, if an LEAS glossary entry did not include the most common way of phrasing an idea, the most common phrasing was added. Third, when an entry included a verb, alternative verb tenses were added. Fourth, when an entry included a word for which there are different forms, the other forms of the word were added (e.g., original entry joy; additional entry joyful). Fifth, if an entry included parenthetical material to clarify meaning, we separated the parenthetical and nonparenthetical material and considered both as possible entries. The changes were made if four of the five expert scorers agreed that the change should be made. The final LEAS Wordlist 2.1 consists of 1,242 word and phrase entries.

Once the Wordlist file and the participants' response data are specified, POES scores the data in three stages, analogous to the three stages of LEAS hand scoring. First, POES scans each item response for words and phrases given in the Wordlist. These words and phrases are called valuables. Each valuable found in the response, along with its score value, is recorded in the valuables list for that item. Next, POES calculates item scores using four different scoring methods (described below). Finally, for each scoring method, the 20 item scores are summed to calculate the total test score. The participants receive four total test scores, one for each scoring method.

POES 1.2.2 uses four methods of calculating item scores for the contents of the valuables list. These methods are summarized in Table 2. The first three methods were used in all samples; the last method was used only in Sample 3, because this method requires separate self and other responses to each question. The first method, All-Sum, calculates the sum of all of the values in the valuables list for an item. This method is the most straightforward procedure and incorporates all of the information given in a response. Because of its simplicity and generality, All-Sum is the scoring method that is most applicable to scoring other open-ended tests, besides the LEAS.

The second method, Highest-4, calculates the sum of the four highest values in the valuables list. For LEAS hand scoring, item scores are based on four values: the two highest values for the participant and the two highest values for the other person. Highest- 4 is a simplification of that idea. It looks at the four highest values, but ignores which person an emotion was attributed to.

The third method, 334, is like hand scoring in that it gives higher scores to responses that include multiple distinct emotion words than to responses in which identical words or phrases are used repeatedly. Specifically, the 334 method searches the valuables list for all valuables with a value of 3 . If these are all identical, an item score of 3 is assigned. If any two valuables are not identical, an item score of 4 
Table 2

POES 1.2.2 Scoring Methods

\begin{tabular}{|c|c|c|}
\hline Name & Description & Example Score \\
\hline All-Sum & Calculates the sum of all of the values in the valuables list for an item. & 11 \\
\hline Highest-4 & Calculates the sum of the four highest values in the valuables list. & 10 \\
\hline 334 & $\begin{array}{l}\text { Searches the valuables list for all valuables with a value of } 3 \text {. If these are all identical, an item score of } 3 \text { is as- } \\
\text { signed. If any two valuables are not identical, an item score of } 4 \text { is given. If there are no valuables with a value } \\
\text { of } 3 \text {, the item score is the maximum value found in the valuables list. }\end{array}$ & 4 \\
\hline 3345 & $\begin{array}{l}\text { Self and other valuables lists are scored separately using the } 334 \text { method. An item score of } 5 \text { is given if the self } \\
\text { and other scores are both } 4 \text {; otherwise, the item score is the maximum of the self and other scores. This method } \\
\text { requires separate areas for self and other responses. }\end{array}$ & 3 \\
\hline
\end{tabular}

Note-The example score is for the response I would be in pain (1) and would probably start crying (2), but I would also be angry (3). My friend would feel a bit guilty (3) about making me cry (2), but would not understand (0) my reaction. The value for each valuable that appears in the LEAS Wordlist is given in parentheses.

is given. If there are no valuables with a value of 3 , the item score is the maximum value found in the valuables list.

The final scoring method, 3345, provides the best emulation of LEAS hand scoring. This method uses the separate responses to the questions "How would you feel?" and "How would the other person feel?" to distinguish between emotions attributed to self and other. In this study, the responses were separated by using two text input boxes. On a paper-administered form, there could be separate areas for responses to the two questions. In either case, this separation only approximates the distinction made during hand scoring, because LEAS responses often attribute emotions to the participant and to the other person in the same sentence. There is no guarantee that respondents enter only self emotions in the "How would you feel?" area and other emotions in the "How would the other person feel?" area.

The 3345 method calculates an item score in three steps. First, self and other valuables lists are created on the basis of the responses to the two questions. Next, these lists are scored separately using the 334 method to obtain self and other scores. Finally, the item score is calculated on the basis of the self and other scores: An item score of 5 is given if the self and other scores are both 4; otherwise, the item score is the maximum of the self and other scores. Because the 334 and 3345 methods are logically closest to hand scoring, we expected them to have higher correlations with hand scoring and more similar correlations with the criterion variables.

The POES 1.2.2 scoring methods follow concrete algorithms and do not attempt to mimic any of the subjective judgments required for hand scoring. In particular, POES does not determine word meaning on the basis of context, does not consider synonyms, and does not actively try to decide whether an emotion is attributed to the participant or to another person. Thus, even the POES scoring methods that most closely mimic hand scoring only approximate human scoring. Thus, the purpose of this study is to evaluate the reliability and validity of these scoring methods. Four different methods were included so that we could assess the value of increasing scoring complexity to more closely mimic hand scoring.

\section{Cerebral Blood Flow}

In Sample 1, we examined the relationship of POES scores to changes in cerebral blood flow due to emotional arousal. We varied the participants' levels of emotional arousal by having each participant complete eight conditions: six picture viewing conditions and two visual fixation conditions. During the six picture viewing conditions, the participants looked at pictures from the International Affective Picture System (Lang, Bradley, \& Cuthbert, 2005). These pictures have been normatively rated on the basis of valence (pleasant-unpleasant) and arousal. In this study, four of the conditions consisted of high- and low-arousal pictures that were pleasant or unpleasant. The two remaining picture-viewing conditions were neutral on valence: One contained human faces in each picture, and the other consisted mostly of scenes and everyday objects. These six picture-viewing conditions were counterbalanced around the first and fifth conditions, which were always visual fixation. During all eight conditions, the participants were told, "Please look at the screen and allow yourself to feel whatever each picture evokes in you." While the participants viewed the screen, functional images were acquired on an Exact HR + PET scanner (Siemens, Knoxville, TN) operating in 3-D mode. See McRae et al. (2008) for details regarding the PET imaging procedures.

To identify those areas of the brain in which changes in blood flow are most highly associated with LEAS scores, image preprocessing and voxel-based analyses were performed using the Statistical Parametric Mapping program. We followed the standard practice of partialling out global blood flow. Details regarding the analyses of LEAS hand scores are given in McRae et al. (2008). In this article, we repeated those analyses, substituting POES scores where McRae et al. used hand scores.

\section{Emotion Expressions}

The Emotion Expressions task assesses recognition of emotion cues in faces (Fine, Izard, Mostow, Trentacosta, \& Ackerman, 2003). The children in Sample 2 viewed 18 facial photos of adults expressing one of six emotions (anger, surprise, sadness, disgust, joy, or fear), taken from Izard's I-M series (Izard, 1971) and the Glenn pictures (Glenn, 1974). These 18 photos were recommended by C. E Izard (personal communication, April 2000). For each photo, the children wrote down what the person was feeling. The response was scored 2 if the correct emotion or a synonym was given, 1 if the emotion was inaccurate but had the correct positive or negative valence (e.g., anger instead of fear), and 0 if both the emotion and valence were incorrect. Internal consistency of the Emotion Expressions task has been reported as $\alpha=.52$ (Schultz \& Izard, 1998). In this study, the internal consistency was $\alpha=.52$.

\section{Emotion Comprehension}

The children in Sample 2 completed an Emotion Comprehension task (Cermele, Ackerman, \& Izard, 1995). This task assesses recognition of emotion cues in situations (Garner, Jones, \& Miner, 1994; Ribordy, Camras, Stefani, \& Spaccarelli, 1988). The children listened to two series of emotionally evocative scenarios while reading along silently. In the 18 scenarios in the first series, the children selected the emotional response of the protagonist from the following list: happy, sad, mad, interested, or ashamed. In the 9 scenarios in the second series, the children selected from a slightly different list of responses: happy, mad, proud, guilty, ashamed, or looking down on someone. As was the case with the Emotion Expressions task, the responses were scored according to accuracy and valence. Internal consistency of the Emotion Comprehension task has been reported as $\alpha=.58$ (Schultz \& Izard, 1998). In this study, internal consistency was $\alpha=.59$.

\section{Vocabulary}

The children in Sample 2 completed the Vocabulary subtest of the WISC-III. In this study, the task was group administered (Barrett, 
Table 3

Internal Consistency

\begin{tabular}{|c|c|c|}
\hline Scoring Method & Coefficient Alpha & $95 \%$ Confidence Interval \\
\hline \multicolumn{3}{|c|}{ Sample 1 (Adult Community Members), Paper LEAS } \\
\hline POES Highest-4 & $.89^{* * \mathrm{a}}$ & {$[.84, .93]$} \\
\hline POES All-Sum & $.89^{* * a}$ & {$[.83, .93]$} \\
\hline POES 334 & $.81^{* * \mathrm{~b}}$ & {$[.72, .88]$} \\
\hline Hand Scoring & $.84^{* * \mathrm{~b}}$ & {$[.77, .90]$} \\
\hline \multicolumn{3}{|c|}{ Sample 2 (Children), Paper LEAS-C } \\
\hline POES Highest-4 & $.78^{* * a}$ & {$[.68, .86]$} \\
\hline POES All-Sum & $.76^{* * \mathrm{~b}}$ & {$[.65, .84]$} \\
\hline POES 334 & $.60^{* * *} \mathrm{c}$ & {$[.41, .74]$} \\
\hline Hand Scoring & $.66^{* * \mathrm{~b}, \mathrm{c}}$ & {$[.51, .78]$} \\
\hline \multicolumn{3}{|c|}{ Sample 3 (Adult Students), Computerized LEAS } \\
\hline POES Highest-4 & $.91^{* * a}$ & {$[.88, .94]$} \\
\hline POES All-Sum & $.92^{* * \mathrm{a}}$ & {$[.89, .94]$} \\
\hline POES 334 & $.79^{* * \mathrm{~b}}$ & {$[.71, .86]$} \\
\hline POES 3345 & $.86^{* * \mathrm{c}}$ & {$[.81, .90]$} \\
\hline Hand Scoring & $.88^{* * \mathrm{c}}$ & {$[.83, .92]$} \\
\hline
\end{tabular}

Note-The values of coefficient alpha were compared with each other using the Pitman technique recommended by Feldt (1980). Coefficients with different superscripted letters were significantly different using $p<$ .05. For example, $.66^{\mathrm{b}, \mathrm{c}}$ was significantly different from $.89^{\mathrm{a}}$ but not from $.81^{\mathrm{b}}$ or $.60^{\mathrm{c}} .95 \%$ confidence intervals were calculated using the Feldt (1965) technique. ${ }^{* *} p<.001$.

Lane, Sechrest, \& Schwartz, 2000; Subic-Wrana, Thomas, Huber, $\&$ Köhle, 2001). Fifteen words were read aloud to the students, the starting point being the one recommended for the lowest age of the participants (Sattler, 1992). As each word was presented, the children wrote down its meaning. The average internal consistency for the Vocabulary subtest is $\alpha=.87$ (Kamphaus, 2005). In this study, the internal consistency of the subtest was $\alpha=.71$.

\section{RESULTS}

\section{Internal Consistency (All Samples)}

For all three samples, the four POES scoring methods had acceptable internal consistencies (see Table 3). For the adult samples, internal consistencies ranged from good $(\alpha=.79)$ to excellent $(\alpha=.92)$. For the child sample, the POES 334 method had barely acceptable internal consistency $(\alpha=.60)$. However, hand scoring also had only acceptable levels of internal consistency in this sample, and the internal consistency of the 334 method was not significantly lower than the internal consistency for the hand scoring.

The POES methods differed in their internal consistencies. The Highest-4 and All-Sum methods had the highest internal consistencies in all three samples. The internal consistencies for the 334 and 3345 methods were the most similar to hand scoring, and in most cases, coefficient alpha for these two POES methods was not significantly different from coefficient alpha for hand scoring.

\section{Correlations of the Four POES Scores With Hand Scoring (All Samples)}

For all three samples, each of the four POES scores had high correlations with hand scoring. This indicates that all of the POES scoring methods tapped the same general construct as hand scoring, thus providing evidence of concurrent validity (see Table 4).
Next, we wanted to determine which POES methods had the highest correlations with hand scoring. We had hypothesized that the 3345 and 334 methods - the methods that were logically most similar to hand scoring - would have higher correlations with hand scoring than would the All-Sum and Highest-4 methods. However, this was not always true, and sometimes the reverse was true. The 334 and Highest- 4 methods appear to be roughly comparable in terms of their correlation with hand scoring.

The 3345 method had a significantly higher correlation with hand scoring than any of the other POES scoring methods in the one sample (adult students) in which it was calculated. Because it was possible to use this scoring method only in the sample in which the computeradministered LEAS was used, this result requires replication before we can be confident that the 3345 method has the highest correlation with hand scoring.

To further examine the similarity of the constructs underlying hand scoring and computer scoring, we corrected these correlations for attenuation due to lack of internal

Table 4

Correlations of POES Total Scores

With Hand Scoring Total Scores

\begin{tabular}{|c|c|c|c|c|}
\hline $\begin{array}{l}\text { POES } \\
\text { Method }\end{array}$ & Correlation & $\begin{array}{c}\text { CI for } \\
\text { Correlation }\end{array}$ & $\begin{array}{c}\text { Correlation } \\
\text { When Corrected } \\
\text { for Attenuation }\end{array}$ & $\begin{array}{c}\text { CI for } \\
\text { Corrected } \\
\text { Correlation }\end{array}$ \\
\hline \multicolumn{5}{|c|}{ Sample 1 (Adult Community Members), Paper LEAS } \\
\hline Highest-4 & $.93^{* * a}$ & {$[.87, .96]$} & $.91^{* *}$ & {$[.66,1.07]$} \\
\hline All-Sum & $.89^{* * \mathrm{~b}}$ & {$[.81, .94]$} & $.91^{* *}$ & {$[.66,1.07]$} \\
\hline 334 & $.92^{* * a, b}$ & {$[.86, .96]$} & $.90^{* *}$ & {$[.60,1.10]$} \\
\hline \multicolumn{5}{|c|}{ Sample 2 (Children), Paper LEAS-C } \\
\hline Highest-4 & $.61^{* * a}$ & {$[.40, .76]$} & $.65^{*}$ & {$[.18,1.02]$} \\
\hline All-Sum & $.61^{* * a}$ & {$[.40, .76]$} & $.65^{*}$ & {$[.18,1.04]$} \\
\hline 334 & $.69^{* * a}$ & {$[.51, .81]$} & $.84^{*}$ & {$[.23,1.34]$} \\
\hline \multicolumn{5}{|c|}{ Sample 3 (Adult Students), Computerized LEAS } \\
\hline Highest-4 & $.80^{* * a}$ & {$[.68, .87]$} & $.87^{* *}$ & {$[.69,1.01]$} \\
\hline All-Sum & $.76^{* * \mathrm{~b}}$ & {$[.64, .85]$} & $.82^{* *}$ & {$[.61, .96]$} \\
\hline 334 & $.80^{* * a}$ & {$[.70, .88]$} & $.97^{* *}$ & {$[.74,1.13]$} \\
\hline 3345 & $.86^{* * \mathrm{c}}$ & {$[.79, .92]$} & $.98^{* *}$ & {$[.81,1.10]$} \\
\hline
\end{tabular}

Note-Correlations with different superscripted letters are significantly different using $p<.05$ using Williams's (1959) T2 statistic. 95\% confidence intervals (CIs) were calculated using the Fisher $r$-to-z transformation described in Howell (2007). We corrected these correlations for attenuation due to lack of internal consistency. Because POES scoring and hand scoring shared the same items, to correct for lack of internal consistency, we used a procedure recommended by L. S. Feldt (personal communication, February, 2006). For each POES method, we first calculated the correlation between Form A for hand scoring and Form B for POES scoring and corrected the correlation using the internal consistencies of those two half-length tests. This provides an unbiased estimate of the correlation when it has been corrected for attenuation due to lack of internal consistency in the two tests. Second, we calculated the correlation for Form B for hand scoring and Form A for POES scoring and corrected the correlation using the internal consistencies of those two half-length tests. Finally, we averaged the two corrected correlations. $95 \%$ CIs were calculated using a similar procedure. First, we calculated the CI for the correlation between Form A for hand scoring and Form B for POES scoring, using the Fisher $r$-to- $z$ method recommended by Howell (2007). We then disattenuated the endpoints. Next, we calculated the CI for the disattenuated correlation between Form B for hand scoring and Form A for POES scoring. Finally, we averaged the endpoints of the two CIs. ${ }^{*} p<.05 .{ }^{* *} p<.001$. 
Table 5

Associations With Cerebral Blood Flow

\begin{tabular}{ccc}
\multicolumn{2}{c}{ Associations With Cerebral Blood Flow } \\
\hline $\begin{array}{c}\text { Scoring } \\
\text { Method }\end{array}$ & \multicolumn{2}{c}{$\begin{array}{c}\text { Area With Highest } \\
\text { Association }\end{array}$} \\
\cline { 2 - 3 } & Coordinates & $z$ Score \\
\hline Highest-4 & $0,12,32$ & $3.41^{* *}$ \\
All-Sum & $0,12,32$ & $3.59^{* *}$ \\
334 & $0,12,32$ & $3.59^{* *}$ \\
Hand Scoring & $0,12,32$ & $4.04^{* *}$ \\
\hline
\end{tabular}

${ }^{* *} p<.001$.

consistency (see Table 4). These corrected correlations were often quite high, and for Sample 3 (adult students), the corrected correlations for the 334 and 3345 methods were close to 1. For the LEAS-C used in Sample 2 (children), the corrected correlations were somewhat low. It may be that the lower emotional awareness of children results in restriction of range, which reduces these correlations.

\section{Associations With Criterion Variables (Sample 1)}

McRae et al. (2008) showed that LEAS hand scores are associated with changes in blood flow in the dACC between the high-arousal and low-arousal conditions, using the data in Sample 1. The purpose of the present analyses was to determine whether a similar area of the dACC would be associated with POES scores. We therefore repeated McRae et al.'s analyses, but we replaced the hand-scored LEAS with the Highest-4, All-Sum, and 334 scores. All three POES scoring methods were significantly related to changes in blood flow between the high- and low-arousal conditions. Moreover, for all three methods, the area with the highest association with POES scores was in Brodmann's area 24 coordinates $0,12,32$ (see Table 5). As was mentioned previously, this cerebral area is associated with awareness and monitoring of emotional experiences (Hutcherson et al., 2005; Ochsner et al., 2002; Taylor et al., 2003).
Associations With Criterion Variables (Sample 2)

Bajgar et al. (2005) correlated hand scores with Emotion Expressions, Emotion Comprehension, and Vocabulary, using the data in Sample 2. The purpose of the present study was to determine whether the correlations for POES scoring would be similar to the correlations reported by Bajgar et al. for hand scoring. We therefore correlated the three POES scores with the three criterion variables. Table 6 shows that all three POES methods had significant (or nearly significant) correlations with each of the three criterion variables. The correlations with emotion expressions and emotion comprehension were moderate, as would be expected because these are related but not identical concepts. The correlations with vocabulary were also moderate, as would be expected because emotional experiences are conveyed through language. These results provide evidence for the validity of all three POES methods.

We wanted to determine which POES scoring methods had the highest correlations. Because the LEAS-C does not provide separate areas for self and other responses, we could not calculate the 3345 method. Of the remaining three methods, the correlations for the 334 method were the most similar to the correlations for hand scoring, as was expected. The Highest-4 method had consistently higher correlations than the other POES methods, but these correlations were not significantly greater than the correlations for the other POES methods.

\section{DISCUSSION}

The LEAS can assist in clinical practice and research. Awareness of emotional experiences is fundamental to individuals' ability to be self-reflective and to relate harmoniously with others. Emotional dysfunctions, such as restricted expression of emotions, difficulties modulating the intensity and duration of emotions, and predominance of emotions such as anger, sadness, or anxiety, are central to a number of Axis I and Axis II disorders

Table 6

Correlations Between LEAS-C and Criterion Variables, Sample 2 (Children)

\begin{tabular}{clcc}
\hline \multirow{2}{*}{ Criterion Variable } & Scoring Method & Correlation & $\begin{array}{c}95 \% \text { Confidence } \\
\text { Interval }\end{array}$ \\
\hline Emotion Expressions & POES Highest-4 & $.46^{* * a}$ & {$[.21, .65]$} \\
& POES All-Sum & $.40^{* \mathrm{~b}}$ & {$[.14, .61]$} \\
& POES 334 & $.29^{* \mathrm{a}, \mathrm{b}, \mathrm{c}}$ & {$[.02, .53]$} \\
& Hand Scoring & $.15^{\mathrm{c}}$ & {$[-.13, .41]$} \\
Emotion Comprehension & POES Highest-4 & $.29^{* \mathrm{a}}$ & {$[.01, .52]$} \\
& POES All-Sum & $.27^{\dagger \mathrm{a}}$ & {$[-.01, .51]$} \\
& POES 334 & $.28^{* \mathrm{a}}$ & {$[.00, .51]$} \\
& Hand Scoring & $.28^{\mathrm{*a}}$ & {$[.00, .51]$} \\
& POES Highest-4 & $.46^{* * \mathrm{a}}$ & {$[.21, .65]$} \\
& POES All-Sum & $.44^{* \mathrm{a}}$ & {$[.19, .64]$} \\
& POES 334 & $.38^{* \mathrm{a}}$ & {$[.12, .60]$} \\
& Hand Scoring & $.31^{* \mathrm{a}}$ & {$[.03, .54]$} \\
\hline
\end{tabular}

Note-Correlations with different superscripted letters were significantly different using $p<.05$ using Williams's (1959) T2 statistic. Confidence intervals for the correlations were calculated using the Fisher $r$-to- $z$ method recommended by Howell (2007). $\quad{ }^{\dagger} p<.10 . \quad{ }^{*} p<.05 . \quad{ }^{* *} p<.001$. 
(American Psychiatric Association, 1994). Because of this, many mental health interventions increase awareness of emotions (Greenberg, 2002; Linehan, 1993) to lay the groundwork for understanding more complex processes, such as the source of emotions, appropriateness of emotions, and the impact of emotions on functioning. Because the LEAS measures the degree of complexity in the cognitive processing of emotion information, it has clinical relevance to understanding individual mental health needs and can facilitate research on the etiology and treatment of clinical disorders.

Research and clinical practice using the LEAS is hampered, unfortunately, because this measure is time consuming to score. Lane et al. (1990) recommended automating the LEAS, which would greatly reduce scoring time and would eliminate the need to evaluate interrater reliability. The purpose of this article was to examine the usefulness of our attempt to automate the LEAS. The results were highly encouraging. The four computer scoring methods resulted in high internal consistencies and high correlations with hand scoring in the adult samples and moderately high internal consistencies and correlations with hand scoring in the child sample. POES scores also demonstrated validity by having moderate correlations with Emotion Expressions, Emotion Comprehension, and Vocabulary, and by being associated with changes in cerebral blood flow in the exact same location as hand scoring. These results suggest that POES scoring could be used instead of hand scoring in situations for which the validity of hand scoring is known. The paper-based adult LEAS has significant validity evidence in a variety of contexts. Research on the validity of the computer-based adult LEAS and the LEAS-C has just begun, but could fruitfully use POES scoring.

Two primary differences were found between the four computer scoring methods that we examined. First, the 334 and 3345 methods had slightly higher correlations with hand scoring, as was expected. Therefore, if researchers or applied psychologists are trying to select a computer scoring method that is as similar as possible to the well-validated hand-scoring method, then the 334 or 3345 methods should be used. Of these two, the 3345 method had a significantly higher correlation with hand scoring and slightly higher internal consistency and is therefore preferred if both scoring methods are an option (i.e., if respondents provided separate answers to the two questions for each item).

The second primary difference was in the reliability and validity evidence. The Highest-4 and All-Sum methods had significantly higher internal consistencies and slightly higher correlations with emotion expressions. Thus, if a test user is trying to select the computer scoring method with the greatest validity evidence, these two methods are recommended. Of these two, the Highest-4 method had significantly higher correlations with hand scoring and emotion expressions and is therefore preferred. Additional validity studies are needed to replicate and extend these findings in order to conclude more definitely which POES method is the most reliable and valid in any particular context. However, the diversity of samples, LEAS versions, and criterion-related variables examined in this article leads us to predict the generalizability of these conclusions.

This article was limited by the small sizes of each of the three samples. Small sample sizes limit statistical power. Although the Type I error rate is controlled by alpha (in this article, we used $\alpha=.05$ ), the Type II error rate can become high. For example, although the Highest-4 method had significantly higher internal consistency than hand scoring in each of the three samples, hand scoring had higher internal consistency than the 334 method in each of the three samples, but none of those differences reached statistical significance. Similarly, the differences between the correlations for the four POES methods rarely reached statistical significance. Because of the small samples sizes, we cannot definitively state which scoring method is the best, even though we were able to provide compelling evidence for the internal consistency, concurrent validity, and criterion-related validity of the POES scoring methods. Future researchers should strive to incorporate larger sample sizes so that we can more clearly determine which scoring methods are preferred. Until future researchers definitively indicate which POES method is the best, all four POES methods should be used when feasible.

Future researchers could use POES to score other constructs. The LEAS Wordlist was designed to score open-ended responses for emotional awareness. In this Wordlist, higher scores are given to emotion words that indicate greater specificity and complexity. By using other wordlists, POES can be used to score other constructs. Thus, POES goes beyond the functionality of LIWC and DAL. LIWC counts the number of words that fall into each linguistic category and allows user-defined lists, but all words within those categories receive identical scores. DAL looks up the scores for each word, but is limited to the two dimensions of pleasantness and activity level. POES combines the best features of each program, by allowing the researcher to use their own wordlists. Moreover, POES goes beyond LIWC and DAL because it includes more than one scoring method. All-Sum is similar to the methods used by LIWC and DAL, but Highest- 4 is also likely to be relevant to many testing circumstances. Finally, POES is more flexible than the other programs, because the detailed score report from POES can be used to produce descriptive statistics, such as the number of times that certain valuables are used or the number of times that a certain value is used. Combined with customcreated Wordlists, this makes POES adaptable to scoring the LEAS for additional constructs and to scoring new open-ended tests. The next revolution in computerized scoring may be an incorporation of user-defined scoring methods. Because POES is distributed under the GNU General Public License, researchers are welcome to modify it to include new scoring methods or user-defined scoring methods. Users are also encouraged to offer suggestions if they do not have the programming skills to make the changes themselves.

Finally, future researchers should explore other ways of using POES to assist scoring. For example, researchers could use POES as an aid in hand scoring, rather than using it as a substitute for hand scoring as we did here. 
A fully trained LEAS scorer could use the detailed score report available from POES to identify words and phrases that need to be scored. Words and phrases that have multiple values in the LEAS glossary could be flagged by being assigned a score of 99 in the Wordlist, and the researcher could determine the appropriate score on the basis of context. However, we cannot recommend the use of POES as an aid in hand scoring until research demonstrates the reliability and validity of scores derived in that manner. Although it is true that scorers sometimes overlook words that occur in the LEAS glossary (and the use of POES would likely prevent that), hand scoring is also based on words that do not occur in the LEAS Glossary, and it is possible that the use of POES would make it more likely that those words would be overlooked unless the Wordlist was made to be truly comprehensive. Research in this area would be valuable.

Taken as a whole, the results of this study indicate that LEAS computer scoring and hand scoring are tapping the same general construct and that computer scoring may be used instead of hand scoring in some contexts. Such use could enlighten and facilitate research on the etiology, nature, and treatment of clinical disorders. In addition, computer scoring could facilitate the measurement of emotional awareness in applied settings, where clinicians, educators, and human resource personnel may not have time to score by hand. Research on LEAS-like scenarios that are specialized for specific disorders (e.g., playground bullying, eating disorders, posttraumatic stress disorder) may further stimulate applied use, and will be facilitated by the speed and flexibility of POES scoring.

\section{AUTHOR NOTE}

Portions of these results were presented at the 2004 conference for the Association for Computing Machinery Special Interest Group on Computer Science Education and the 2004 and 2007 conferences of the Western Psychological Association. Correspondence concerning this article should be addressed to K. A. Barchard, University of Nevada, Las Vegas, 4505 Maryland Parkway, Las Vegas, NV 89154-5030 (e-mail: barchard@unlv.nevada.edu)

\section{REFERENCES}

Ali, M., \& Levine, T. (2008). The language of truthful and deceptive denials and confessions. Communication Reports, 21, 82-91. doi: $10.1080 / 08934210802381862$

American Psychiatric Association (1994). Diagnostic and statistical manual of mental disorders: DSM-IV (4th ed.). Washington, DC: Author.

Bajgar, J., Ciarrochi, J., Lane, R., \& Deane, F. P. (2005). Development of the Levels of Emotional Awareness Scale for Children (LEAS-C). British Journal of Developmental Psychology, 23, 569586. doi:10.1348/026151005X35417

BARCHARD, K. A. (2006). LEAS Wordlist 2.1. File to be used with Program for Open-Ended Scoring to allow scoring of the Levels of Emotional Awareness Scale. Available from K. A. Barchard, Department of Psychology, University of Nevada, Las Vegas, 4505 Maryland Parkway, Las Vegas, NV 89154-5030 (e-mail: barchard@unlv.nevada.edu).

Barrett, L. F., Lane, R. D., Sechrest, L., \& Schwartz, G. E. (2000). Sex differences in emotional awareness. Personality \& Social Psychology Bulletin, 26, 1027-1035. doi:10.1177/01461672002611001

Berthoz, S., Ouhayoun, B., Parage, N., Kirzenbaum, M., Bourgey, M., \& Allilaire, J.-F. (2000). Étude préliminaire des niveaux de conscience émotionnelle chez des patients déprimés et des contrôles [Preliminary study of the levels of emotional awareness in depressed patients and controls]. Annales Médico-Psychologiques, 158, 665-672.

Bydlowski, S., Corcos, M., Jeammet, P., Paterniti, S., Berthoz, S., LAURIER, C., ET AL. (2005). Emotion-processing deficits in eating disorders. International Journal of Eating Disorders, 37, 321-329. doi:10.1002/eat.20132

Carroll, D. W. (2007). Patterns of student writing in a critical thinking course: A quantitative analysis. Assessing Writing, 12, 213-227. doi:10.1016/j.asw.2008.02.001

Cermele, J. A., Ackerman, B. P., \& Izard, C. E. (1995). Children's emotion situation knowledge. Unpublished manuscript, University of Delaware, Newark.

Ciarrochi, J., Caputi, P., \& Mayer, J. D. (2003). The distinctiveness and utility of a measure of trait emotional awareness. Personality \& Individual Differences, 34, 1477-1490. doi:10.1016/S0191 -8869(02)00129-0

Donges, U.-S., Kersting, A., Dannlowski, U., Lalee-Mentzel, J., Arolt, V., \& Suslow, T. (2005). Reduced awareness of others' emotions in unipolar depressed patients. Journal of Nervous \& Mental Disease, 193, 331-337. doi:10.1097/01.nmd.0000161683.02482.19

DuBoIs, S. L. (1997). Gender differences in the emotional tone of written sexual fantasies. Canadian Journal of Human Sexuality, 6, 307-315.

FeLDT, L. S. (1965). The approximate sampling distribution of KuderRichardson reliability coefficient twenty. Psychometrika, 30, 357370. doi:10.1007/BF02289499

FELDT, L. S. (1980). A test of the hypothesis that Cronbach's alpha reliability coefficient is the same for two tests administered to the same sample. Psychometrika, 45, 99-105. doi:10.1007/BF02293600

Fine, S. E., Izard, C. E., Mostow, A. J., Trentacosta, C. J., \& AcKERMAN, B. P. (2003). First grade emotion knowledge as a predictor of fifth grade self-reported internalizing behaviors in children from economically disadvantaged families. Development \& Psychopathology, 15, 331-342. doi:10.1017/S095457940300018X

FlaVell, J. H. (1963). The developmental psychology of Jean Piaget New York: Van Nostrand.

Free Software Foundation (1991). GNU General Public License (Version 2). Retrieved December 29, 2009, from www.gnu.org/ licenses/old-licenses/gpl-2.0.html

Frewen, P., Lane, R. D., Neufeld, R. W. J., Densmore, M., SteVENS, T., \& Lanius, R. (2008). Neural correlates of levels of emotional awareness during trauma script-imagery in posttraumatic stress disorder. Psychosomatic Medicine, 70, 27-31. doi:10.1097/ PSY.0b013e31815f66d4

Garner, P. W., Jones, D. C., \& Miner, J. L. (1994). Social competence among low-income preschoolers: Emotion socialization practices and social cognitive correlates. Child Development, 65, 622-637. doi:10.2307/1131405

GLENN, R. (1974). The Glenn pictures: Afro American faces of emotion. Bloomington, IN: Indiana University.

GrEenBerg, L. S. (2002). Emotion-focused therapy: Coaching clients to work through their feelings. Washington, DC: American Psychological Association.

Howell, D. C. (2007). Statistical methods for psychology (6th ed.). Belmont, CA: Thomson Wadsworth.

Hutcherson, C. A., Goldin, P. R., Ochsner, K. N., Gabrieli, J. D. E. Feldman Barrett, L., \& Gross, J. J. (2005). Attention and emotion: Does rating emotion alter neural responses to amusing and sad films? NeuroImage, 27, 656-668. doi:10.1016/j.neuroimage.2005.04.028

IzARD, C. E. (1971). The face of emotion. New York: Appleton-CenturyCrofts.

Jouanne, C., Edel, Y., \& Carton, S. (2005). Déficits émotionnels chez des patients polytoxicomanes [Emotional deficits among multiple substance abusers]. Annales Médico-Psychologiques, 163, 625-630.

Kamphaus, R. W. (2005). Clinical assessment of child and adolescent intelligence (2nd ed.). New York: Springer.

LANE, R. D. (1991). LEAS scoring manual and glossary. Unpublished manuscript. Available from R. D. Lane, General Clinical Research Center, University of Arizona, PO Box 245002, Tucson, AZ 85724-5002.

LANE, R. D. (2000). Levels of emotional awareness: Neurological, psychological, and social perspectives. In R. Bar-On \& J. D. A. Parker (Eds.), The handbook of emotional intelligence: Theory, development, assessment and application at home, school, and in the workplace (pp. 171-191). San Francisco: Jossey-Bass. 
Lane, R. D., Quinlan, D. M., Schwartz, G. E., Walker, P. A., \& Zeitlan, S. B. (1990). The Levels of Emotional Awareness Scale: A cognitive-developmental measure of emotion. Journal of Personality Assessment, 55, 124-134. doi:10.1207/s15327752jpa5501\&2_12

Lane, R. D., Reiman, E. M., Axelrod, B., Yun, L.-S., Holmes, A., \& Schwartz, G. E. (1998). Neural correlates of levels of emotional awareness: Evidence of an interaction between emotion and attention in the anterior cingulate cortex. Journal of Cognitive Neuroscience, 10, 525-535. doi:10.1162/089892998562924

LANE, R. D., \& Schwartz, G. E. (1987). Levels of emotional awareness: A cognitive-developmental theory and its application to psychopathology. American Journal of Psychiatry, 144, 133-143.

Lane, R. D., Sechrest, L., Reidel, R., Weldon, V., Kaszniak, A., \& Schwartz, G. E. (1996). Impaired verbal and nonverbal emotion recognition in alexithymia. Psychosomatic Medicine, 58, 203-210.

Lane, R. D., Sechrest, L., \& Riedel, R. (1998). Sociodemographic correlates of alexithymia. Comprehensive Psychiatry, 39, 377-385. doi:10.1016/S0010-440X(98)90051-7

Lane, R. D., Sechrest, L., Riedel, R., Shapiro, D. E., \& Kaszniak, A. W. (2000). Pervasive emotion recognition deficit common to alexithymia and the repressive coping style. Psychosomatic Medicine, 62, 492-501.

Lang, P. J., Bradley, M. M., \& Cuthbert, B. N. (2005). International affective picture system (IAPS): Affective ratings of pictures and instruction manual (Tech. Rep. A-6). University of Florida, Gainesville.

LARKEY, L. S. (1998). Automatic essay grading using text categorization techniques. In W. B. Croft, A. Moffat, C. J. Van Riisbergen, R. Wilkinson, \& J. Zobel (Eds.), Proceedings of the 21st Annual International ACM SIGIR Conference on Research and Development in Information Retrieval (pp. 90-95). New York: ACM Press. doi:10.1145/290941.290965

LeAF, D. E., \& Barchard, K. A. (2002). LEAS Wordlist 1.0. File to be used with Program for Open-Ended Scoring to allow scoring of the Levels of Emotional Awareness Scale. Available from K. A. Barchard, Department of Psychology, University of Nevada, Las Vegas, 4505 Maryland Parkway, Las Vegas, NV 89154-5030 (e-mail: barchard@ unlv.nevada.edu).

LeAF, D. E., \& Barchard, K. A. (2006). Program for Open-Ended Scoring [POES] (Version 1.2.2). [Unpublished computer software]. Available from K. A. Barchard, Department of Psychology, University of Nevada, Las Vegas, 4505 Maryland Parkway, Las Vegas, NV 891545030 (e-mail: barchard@unlv.nevada.edu).

Levine, D., Marziali, E., \& Hood, J. (1997). Emotion processing in borderline personality disorders. Journal of Nervous \& Mental Disease, 185, 240-246. doi:10.1097/00005053-199704000-00004

LineHAN, M. M. (1993). Skills training manual for treating borderline personality disorder. New York: Guilford.

McRae, K., Reiman, E. M., Fort, C. L., Chen, K., \& Lane, R. D. (2008). Association between trait emotional awareness and dorsal anterior cingulate activity during emotion is arousal-dependent. NeuroImage, 41, 648-655. doi:10.1016/j.neuroimage.2008.02.030

Mossholder, K. W., Settoon, R. P., Harris, S. G., \& Armenakis, A. A. (1995). Measuring emotion in open-ended survey responses: An application of textual data analysis. Journal of Management, 21, 335-355. doi:10.1016/0149-2063(95)90061-6

Ochsner, K. N., Bunge, S. A., Gross, J. J., \& Gabrieli, J. D. E. (2002). Rethinking feelings: An fMRI study of the cognitive regulation of emotion. Journal of Cognitive Neuroscience, 14, 1215-1229. doi:10.1162/089892902760807212

Pennebaker, J. W. (1997). Writing about emotional experiences as a therapeutic process. Psychological Science, 8, 162-166. doi:10.1111/j.1467-9280.1997.tb00403.x

Pennebaker, J. W., Chung, C. K., Ireland, M., Gonzales, A., \& Bоотн, R. J. (2008). The development and psychometric properties of LIWC2007. Retrieved December 29, 2009, from www.liwc.net/ LIWC2007LanguageManual.pdf.

Ribordy, S. C., Camras, L. A., Stefani, R., \& Spaccarelli, S. (1988). Vignettes for emotion recognition research and affective therapy with children. Journal of Clinical Child Psychology, 17, 322-325. doi:10.1207/s15374424jccp1704_4

Rose, D. (2004). Theory and treatment of alexithymia: An affect theory perspective. Dissertation Abstracts International, 64(8), 4060B.

Russell, J. A. (1980). A circumplex model of affect. Journal of Personality \& Social Psychology, 39, 1161-1178. doi:10.1037/h0077714

SATTLER, J. M. (1992). Assessment of children: WISC-III and WPPSI-R supplement. La Mesa, CA: Author.

Schultz, D., \& IZARD, C. E. (1998). [The assessment of children's emotion skills: Facial expressions subtest]. Unpublished raw data.

Shermis, M. D., \& BursteIn, J. (EDS.) (2003). Automated essay scoring: A cross-disciplinary perspective. Mahwah, $\mathrm{NJ}$ : Erlbaum.

Simson, U., Martin, K., Schäfer, R., Franz, M., \& Janssen, P. (2006). Veränderung der Wahrnehmung von Emotionen im Verlauf stationärer psychotherapeutischer Behandlung [Changes in alexithymia and emotional awareness during psychotherapeutic inpatient treatment]. Psychotherapie, Psychosomatik, Medizinische Psychologie, 56, 376-382. doi:10.1055/s-2006-940127

Subic-Wrana, C., Bruder, S., Thomas, W., Gaus, E., Merkle, W. \& KöHLE, K. (2002). Verteilung des Persönlichkeitsmerkmals Alexithymie bei Patienten in stationärer psychosomatischer Behandlunggemessen mit TAS-20 und LEAS [Distribution of alexithymia as a personality trait in psychosomatically ill in-patients - measured with TAS-20 and LEAS]. Psychotherapie, Psychosomatik, Medizinische Psychologie, 52, 454-460. doi:10.1055/s-2002-35281

Subic-Wrana, C., Bruder, S., Thomas, W., Lane, R. D., \& KöHle, K. (2005). Emotional awareness deficits in inpatients of a psychosomatic ward: A comparison of two different measures of alexithymia. Psychosomatic Medicine, 67, 483-489. doi:10.1097/01.psy.0000160461.19239.13

Subic-Wrana, C., Thomas, W., Huber, M., \& KöHle, K. (2001). Levels of Emotional Awareness Scale (LEAS): Die deutsche Version eines neuen Alexithymietests [The German version of a new alexithymia test]. Psychotherapeut, 46, 176-181. doi:10.1007/s002780100157

TaYlor, S. F., Phan, K. L., Decker, L. R., \& Liberzon, I. (2003). Subjective rating of emotionally salient stimuli modulates neural activity. NeuroImage, 18, 650-659. doi:10.1016/S1053-8119(02)00051-4

Valenti, S., Neri, F., \& Cucchiarelli, A. (2003). An overview of current research on automated essay grading. Journal of Information Technology Education, 2, 319-330.

WALGREN, M. B. (1996). Alexithymia and levels of emotional development in a substance abuse population. Dissertation Abstracts International, 56(8), 3065A.

Waller, E., \& SCHEIDT, C. E. (2004). Somatoform disorders as disorders of affect regulation: A study comparing the TAS-20 with nonself-report measures of alexithymia. Journal of Psychosomatic Research, 57, 239-247. doi:10.1016/S0022-3999(03)00613-5

WeChSLER, D. (1991). Wechsler Intelligence Scale for Children-Third Edition (WISC-III). San Antonio: Harcourt Assessment.

WhisseLl, C. M. (1989). The dictionary of affect in language. In R. Plutchik \& H. Kellerman (Eds.), The measurement of emotion (Emotion: Theory, research and experience, Vol. 4, pp. 113-131). San Diego: Academic Press.

Whissell, C. [M.] (2008). Emotional fluctuations in Bob Dylan's lyrics measured by the dictionary of affect accompany events and phases in his life. Psychological Reports, 102, 469-483. doi:10.2466/ PR0.102.2.469-483

WiLliams, E. J. (1959). The comparison of regression variables. Journal of the Royal Statistical Society: Series B, 21, 396-399.

YANG, Y., Buckendahl, C. W., Juszkiewicz, P. J., \& Bhola, D. S. (2002). A review of strategies for validating computer-automated scoring. Applied Measurement in Education, 15, 391-412. doi:10.1207/ S15324818AME1504_04

Zwaal, C., Prkachin, K. M., Husted, J., \& Stones, M. (2003). Components of hostility and verbal communication of emotion. Psychology \& Health, 18, 261-273. doi:10.1080/0887044021000058067

(Manuscript received May 13, 2009;

revision accepted for publication December 26, 2009.) 